\title{
Expectativas dos estudantes de Odontologia quanto ao futuro profissional
}

\author{
Milton Duque Marques*; Camila Agra Souza*; Carolina Thaiza Costa Pazos **; Debora Soares do Amaral ${ }^{* *}$; \\ Everton Gomes Vieira*; Wagna Cavalcanti Siqueira Campos**; Elaine Judite de Amorim Carvalho****
}

\footnotetext{
* Acadêmico do Curso de Odontologia da Universidade Federal de Pernambuco

** Cirurgiã-Dentista pela Universidade Federal de Pernambuco

*** Professora Adjunta do Departamento de Clínica e Odontologia Preventiva da Universidade Federal de Pernambuco
}

\section{RESUMO}

Este trabalho teve por objetivo conhecer as expectativas, projetos profissionais e projeções de êxito nas atividades laborais futuras dos estudantes de odontologia da Universidade Federal de Pernambuco. O estudo foi realizado no âmbito da própria universidade, nas dependências do curso de odontologia. A população de estudo foi de estudantes do $1^{\circ}$ período diurno e noturno $(n=43), 5^{\circ}$ período $(n=41)$ e $10^{\circ}$ período $(n=21)$. A maioria dos estudantes entrevistados consistia de jovens, solteiros $(99 \%)$ e do sexo feminino $(65,7 \%)$, com renda familiar de até $\mathrm{R} \$ 5.100,00$ ao mês $(80,9 \%)$. Observou-se que $83,8 \%$ optaram por estudar odontologia por se identificar com a profissão. Os alunos também reconheceram o padrão de qualidade das Instituições Federais de Ensino Superior e este foi o motivo de escolha pela universidade $(74,3 \%)$. Não houve diferenças quanto a ingressar futuramente no mercado laboral público ou privado, sendo ambas opções viáveis na concepção dos entrevistados. A promoção de saúde foi o fator relatado como principal fonte de realização profissional $(71,4 \%)$, especialmente para os alunos de períodos iniciais do curso $(\mathrm{p}=0,026)$. A estimativa inicial de renda está dentro dos limites de renda familiar atual da maioria dos entrevistados $(59 \%)$ e $98,1 \%$ pretendem fazer curso de pós-graduação, tendo nas especializações o objetivo mais comum $(78,6 \%)$. Especialidades como cirurgia bucomaxilofacial, ortodontia e implantodontia serão as mais procuradas.

Descritores: Avaliação. Educação Superior. Odontologia.

\section{INTRODUÇÃO}

Para muitos jovens adultos, o fim de um curso universitário significa a promessa de uma nova fase de vida, marcada pelo início do exercício da profissão escolhida. No entanto, um dos principais problemas com os quais os recém-formados se deparam é a dificuldade de ingressar no mercado de trabalho de suas profissões. $^{1}$ 
Os recém ingressos como estudantes de graduação nas Instituições de Ensino Superior possuem uma visão mais positiva quanto à sua formação quando comparados aos que se encontram nos diversos períodos do curso, as opiniões sobre a eficiência da formação para o mercado são divididas. ${ }^{2,3}$

Sabe-se que a conquista de espaço no mercado não depende apenas de um diploma, mas também de características pessoais, competências específicas, redes de relações e capacidade de ajustar-se a diferentes demandas de trabalho. ${ }^{1}$

Uma parte dos formandos espera trabalhar como assalariados e pretende continuar seus estudos após o início das atividades profissionais. Portanto, a procura da especialização por parte de muitos cirurgiõesdentistas pode estar relacionada à busca por reconhecimento profissional, enquanto a clínica geral é considerada uma prática de menor prestígio para recém-formados. ${ }^{3}$

Ainda que se considerem as dificuldades iniciais que os egressos enfrentam, as expectativas são as mais promissoras possíveis, desde que se garanta a continuidade de estudos, seja em especializações ou em cursos de atualização. Apesar da insegurança que possa haver no início da profissão, os alunos acreditam que estão preparados, embora existam dificuldades em determinadas áreas pouco exploradas clinicamente. ${ }^{4}$

Desta forma, o presente trabalho tem por objetivo conhecer as expectativas, projetos profissionais e projeções de êxito em atividades laborais dos alunos do Curso de Odontologia da Universidade Federal de Pernambuco.

\section{METODOLOGIA}

Este estudo, caracterizado como descritivo transversal, foi submetido ao Comitê de Ética em Pesquisa em Seres Humanos da
UFPE, tendo sido aprovado pelo parecer CAAE 00695712.4.0000.5208 e realizado na Universidade Federal de Pernambuco (UFPE), no âmbito do Curso de Odontologia. A população do estudo foi composta por 150 indivíduos matriculados no curso, divididos nos seguintes grupos:

G1a: 30 estudantes matriculados no $1^{\circ}$ período do curso diurno, critério de escolha aleatória pelos números pares da lista de chamada;

G1b: 20 estudantes matriculados no $1^{\circ}$ período do curso noturno, critério de escolha aleatória pelos números pares da lista de chamada;

G2: 50 estudantes matriculados no $5^{\circ}$ período, a totalidade da turma única; e

G3: 50 estudantes matriculados no $10^{\circ}$ período, a totalidade da turma única.

A coleta de dados foi realizada no período de novembro de 2012 a abril de 2013 e foi iniciada com a visita dos pesquisadores aos ambientes de convivência dos estudantes para convidá-los a participar da pesquisa. Com a aceitação do convite, foi feita uma explicação oral dos objetivos, procedimentos, riscos e benefícios da pesquisa, sendo solicitada a leitura e assinatura do Termo de Consentimento Livre e Esclarecido - TCLE. Depois de assinar o termo, os estudantes responderam a um questionário desenhado especificamente para este estudo, como forma de avaliar as expectativas quanto ao seu futuro profissional.

Os dados foram tabulados e analisados utilizando-se os softwares Microsoft Excel e SPSS versão 21. Foi realizada uma análise do tipo descritiva das informações contidas nos questionários e ainda o teste Qui-Quadrado de Pearson e teste Exato de Fisher.

\section{RESULTADOS}

A faixa etária dos 105 estudantes entrevistados variou de 18 a 50 anos, com média de 21 anos. A maioria era do sexo 
feminino $(n=69,65,7 \%)$ e $36(34,3 \%)$ eram do sexo masculino. Mais de $50 \%$ dos entrevistados relatavam renda familiar superior a $\mathrm{R} \$$ $5.000,00 ; 104$ (99\%) eram solteiros e $1(1 \%)$ divorciado (tabela 1).

A distribuição dos estudantes segundo o semestre letivo em curso se configurou da seguinte maneira: $43(41 \%)$ eram do $1^{\circ}$ período, $41(39 \%)$ eram do $5^{\circ}$ período e $21(20 \%)$ do $10^{\circ}$ período (tabela 1).

Quando perguntados sobre a razão da escolha do curso, mais de $60 \%$ optaram pela carreira de odontologia por identificação com a profissão. Mais de $70 \%$ dos estudantes afirmaram ter escolhido a UFPE pela qualidade de ensino como instituição federal (tabela 2).

Quanto ao futuro profissional e o ambiente em que desejaria trabalhar, houve equiparação entre o serviço público e o privado, sendo que $13 \%$ desejavam seguir carreira acadêmica. $O$ principal fator de realização profissional apontado pelos entrevistados $(71,4 \%)$ foi a possibilidade de promover a saúde. A pretensão salarial inicial variou de $\mathrm{R} \$$ $2.500,00$ a $\mathrm{R} \$ 5.000,00$ para $59 \%$ dos pesquisados (tabela 2).

Tabela 1 - Distribuição dos pesquisados segundo o perfil sociodemográfico.

$\begin{array}{lll}\text { Variável } & \text { n }\end{array}$

\section{Faixa etária}

Até 20 anos

\section{Sexo}

Masculino

\section{Estado civil}

Solteiro

Separado/ Divorciado

$\begin{array}{ll}104 & 99,0 \\ 1 & 1,0\end{array}$

\section{Renda familiar}

$>\mathrm{R} \$ 10.201,00$

$\mathrm{R} \$ 5.101,00$ a $10.200,00$

$\mathrm{R} \$ 2.041,00$ a $5,100,00$

\section{Período}


Em relação à educação continuada, 98\% especializações como Cirurgia Bucomados estudantes pretende fazer pós-graduação, xilofacial $(28,2 \%)$, Ortodontia $(19,4 \%)$ e sendo a sua distribuição maior para as Implantodontia (17,5\%) (tabela 2).

Tabela 2 - Distribuição dos pesquisados segundo as questões relacionadas à expectativa profissional no curso de odontologia da UFPE

\begin{tabular}{|c|c|c|}
\hline Variável & $\mathbf{n}$ & $\%$ \\
\hline \multicolumn{3}{|l|}{ Principal motivo pela escolha do curso de odontologia: } \\
\hline Sempre quis estudar odontologia & 21 & 20,0 \\
\hline Identificação com a profissão & 67 & 63,8 \\
\hline Influência da família & 8 & 7,6 \\
\hline Pela garantia de realização financeira & 3 & 2,9 \\
\hline Outro & 6 & 5,7 \\
\hline TOTAL & 105 & 100,0 \\
\hline \multicolumn{3}{|l|}{ Opção pela UFPE foi motivada principalmente por: } \\
\hline Melhor qualidade das instituições de ensino federais & 78 & 74,3 \\
\hline Caráter gratuito da instituição & 23 & 21,9 \\
\hline Professores mais qualificados & 10 & 9,5 \\
\hline Facilidade de participar de projetos de iniciação científica com bolsas de estudos & 4 & 3,8 \\
\hline \multicolumn{3}{|l|}{ Expectativa quanto ao futuro profissional } \\
\hline Ser dentista do serviço público & 86 & 81,9 \\
\hline Trabalhar em seu consultório privado & 85 & 81,0 \\
\hline Carreira acadêmica & 13 & 12,4 \\
\hline Serviço militar & 03 & 2,9 \\
\hline Ter um emprego em uma clínica particular & 01 & 1,0 \\
\hline Serviço hospitalar & 01 & 1,0 \\
\hline Empresário do ramo, sem exercer diretamente & 01 & 1,0 \\
\hline \multicolumn{3}{|l|}{ Principal aspecto de importância para sua realização profissional } \\
\hline Promover saúde & 75 & 71,4 \\
\hline Ganhar bem & 43 & 41,0 \\
\hline Ser famoso & 01 & 1,0 \\
\hline Trabalhar bem & 01 & 1,0 \\
\hline $\mathbf{B A S E}^{(\mathbf{1})}$ & 105 & \\
\hline \multicolumn{3}{|l|}{ Estimativa inicial da renda } \\
\hline $\mathrm{R} \$ 1000$ a 2500 & 24 & 22,9 \\
\hline $\mathrm{R} \$>2500$ a 5000 & 62 & 59,0 \\
\hline $\mathrm{R} \$>5000$ a 10000 & 11 & 10,5 \\
\hline Não informado & 08 & 7,6 \\
\hline TOTAL & 105 & 100,0 \\
\hline \multicolumn{3}{|l|}{ Pós-Graduação } \\
\hline Sim & 103 & 98,1 \\
\hline Não & 2 & 1,9 \\
\hline TOTAL & 105 & 100,0 \\
\hline \multicolumn{3}{|l|}{ Tipo de pós-graduação } \\
\hline Especialização & 81 & 78,6 \\
\hline Mestrado & 17 & 16,5 \\
\hline Doutorado & 22 & 21,4 \\
\hline Não informado & 7 & 6,8 \\
\hline $\mathbf{B A S E} \mathbf{E}^{(\mathbf{1})}$ & 103 & \\
\hline
\end{tabular}


Tabela 2 - continuação

\begin{tabular}{lll}
\hline Variável & $\mathbf{n}$ & \% \\
\hline Área da pós-graduação & 29 & 28,2 \\
Cirurgia bucomaxilofacial & 20 & 19,4 \\
Ortodontia & 18 & 17,5 \\
Implantodontia & 9 & 8,7 \\
Odontopediatria & 9 & 8,7 \\
Prótese & 8 & 7,8 \\
Endodontia & 7 & 6,8 \\
Patologia oral & 6 & 5,8 \\
Dentística & 4 & 3,9 \\
Saúde coletiva & 4 & 3,9 \\
Periodontia & 4 & 2,9 \\
Odontologia preventiva e social & 3 & 2,9 \\
Radiologia & 3 & 1,9 \\
Odontologia legal & 2 & 1,0 \\
Outra & 2 & 1,0 \\
Odontogeriartria & 1 & 1,0 \\
Empreendedorismo & 1 & -
\end{tabular}

(1): Considerando que um mesmo pesquisado tenha citado mais de uma alternativa, registra-se a base para o cálculo dos percentuais e não o total.

Quando avaliadas questões relacionadas à expectativa profissional segundo o período, não foram encontradas associações estatisticamente significativas entre as variáveis "principal motivo pela escolha do curso de odontologia", "melhor qualidade das instituições federais de ensino superior", "caráter gratuito das instituições de ensino superior", "expectativa quanto ao futuro profissional" e "estimativa inicial de renda" (tabela 3). Em contrapartida, quando a distribuição por períodos foi comparada com a variável de escolha pela UFPE pela melhor qualificação do corpo docente, observou-se que quanto mais avançado era o período letivo, mais foram as respostas positivas neste quesito (tabela 3).

Na questão de realização profissional, a variável "promoção de saúde foi assinalada preferencialmente pelos estudantes do $1^{\circ}$ período quando comparados aos estudantes mais avançados na carreira universitária". De maneira inversa, quando inquiridos sobre "ganhar bem" como principal fator de realização profissional, os estudantes do $10^{\circ}$ período responderam positivamente a esta pergunta com diferenças significativas em relação aos períodos mais iniciais do curso (tabela 3).

\section{DISCUSSÃO}

Vivemos uma época de grandes demandas sociais e amplos questionamentos em relação ao perfil profissional que as Instituições de Ensino Superior (IES) devem lançar no mercado de trabalho brasileiro. A exemplo de outras IES, a UFPE passou recentemente por uma reforma curricular, vivendo a situação da coexistência de dois perfis curriculares. A principal diferença no 
Tabela 3 - Avaliação das questões relacionadas à expectativa profissional no curso de odontologia da UFPE segundo o período.

\begin{tabular}{|c|c|c|c|c|c|c|c|c|c|}
\hline \multirow{3}{*}{ Variável } & \multicolumn{9}{|c|}{ Período } \\
\hline & \multicolumn{2}{|c|}{ 1o } & \multicolumn{2}{|l|}{ 5o } & \multicolumn{2}{|c|}{$10^{0}$} & \multicolumn{2}{|c|}{ Grupo Total } & \multirow[t]{2}{*}{ Valor de $\mathbf{p}$} \\
\hline & $\mathrm{n}$ & $\%$ & $\mathrm{n}$ & $\%$ & $\mathrm{n}$ & $\%$ & $\mathrm{n}$ & $\%$ & \\
\hline \multicolumn{10}{|c|}{ Principal motivo pela escolha do curso de odontologia: } \\
\hline Sempre quis estudar odontologia & 8 & 18,6 & 12 & 29,3 & 1 & 4,8 & 21 & 20,0 & \multirow[t]{4}{*}{$\mathrm{p}^{(1)}=0,148$} \\
\hline Identificação com a profissão & 30 & 69,8 & 23 & 56,1 & 14 & 66,7 & 67 & 63,8 & \\
\hline Influência da família & 2 & 4,7 & 2 & 4,9 & 4 & 19,0 & 8 & 7,6 & \\
\hline Outro & 3 & 7,0 & 4 & 9,8 & 2 & 9,5 & 9 & 8,6 & \\
\hline \multicolumn{10}{|c|}{ Opção pela UFPE foi motivada principalmente por: } \\
\hline \multicolumn{10}{|c|}{ Melhor qualidade das instituições de ensino federais } \\
\hline Sim & 31 & 72,1 & 34 & 82,9 & 13 & 61,9 & 78 & 74,3 & \multirow[t]{2}{*}{$\mathrm{p}^{(2)}=0,183$} \\
\hline Não & 12 & 27,9 & 7 & 17,1 & 8 & 38,1 & 27 & 25,7 & \\
\hline \multicolumn{10}{|l|}{ Caráter gratuito da instituição } \\
\hline Sim & 9 & 20,9 & 8 & 19,5 & 6 & 28,6 & 23 & 21,9 & \multirow[t]{2}{*}{$\mathrm{p}^{(2)}=0,702$} \\
\hline Não & 34 & 79,1 & 33 & 80,5 & 15 & 71,4 & 82 & 78,1 & \\
\hline \multicolumn{10}{|l|}{ Professores mais qualificados } \\
\hline Sim & - & - & 7 & 17,1 & 3 & 14,3 & 10 & 9,5 & \multirow[t]{2}{*}{$\mathrm{p}^{(1)}=0,009^{*}$} \\
\hline Não & 43 & 100,0 & 34 & 82,9 & 18 & 85,7 & 95 & 90,5 & \\
\hline \multicolumn{10}{|c|}{$\begin{array}{l}\text { Expectativa quanto ao futuro profissional } \\
\text { Ser dentista do servico público }\end{array}$} \\
\hline Sim & 35 & 81,4 & 36 & 87,8 & 15 & 71,4 & 86 & 81,9 & \multirow[t]{2}{*}{$\mathrm{p}^{(2)}=0,283$} \\
\hline Não & 8 & 18,6 & 5 & 12,2 & 6 & 28,6 & 19 & 18,1 & \\
\hline \multicolumn{10}{|c|}{ Trabalhar em seu consultório privado } \\
\hline Sim & 32 & 74,4 & 34 & 82,9 & 19 & 90,5 & 85 & 81,0 & $\mathrm{p}^{(2)}=0,282$ \\
\hline Não & 11 & 25,6 & 7 & 17,1 & 2 & 9,5 & 20 & 19,0 & \\
\hline Carreira acadêmica & & & & & & & & & \\
\hline Sim & 7 & 16,3 & 6 & 14,6 & - & - & 13 & 12,4 & $\mathrm{p}^{(2)}=0,152$ \\
\hline Não & 36 & 83,7 & 35 & 85,4 & 21 & 100,0 & 92 & 87,6 & \\
\hline Principal aspecto de importância & ua real & ação pr & fissio & & & & & & \\
\hline Promover saúde & & & & & & & & & \\
\hline Sim & 33 & 76,7 & 32 & 78,0 & 10 & 47,6 & 75 & 71,4 & $\mathrm{p}^{(2)}=0,026^{*}$ \\
\hline Não & 10 & 23,3 & 9 & 22,0 & 11 & 52,4 & 30 & 28,6 & \\
\hline Ganhar bem & & & & & & & & & \\
\hline Sim & 11 & 25,6 & 21 & 51,2 & 11 & 52,4 & 43 & 41,0 & $\mathrm{p}^{(2)}=0,028^{*}$ \\
\hline Não & 32 & 74,4 & 20 & 48,8 & 10 & 47,6 & 62 & 59,0 & \\
\hline TOTAL & 43 & 100,0 & 41 & 100,0 & 21 & 100,0 & 105 & 100,0 & \\
\hline Estimativa inicial da renda & & & & & & & & & \\
\hline $\mathrm{R} \$ 1000$ a 2500 & 14 & 35,0 & 7 & 18,4 & 3 & 15,8 & 24 & 24,7 & $\mathrm{p}^{(1)}=0,190$ \\
\hline $\mathrm{R} \$>2500$ a 5000 & 24 & 60,0 & 24 & 63,2 & 14 & 73,7 & 62 & 63,9 & \\
\hline $\mathrm{R} \$>5000$ a 10000 & 2 & 5,0 & 7 & 18,4 & 2 & 10,5 & 11 & 11,3 & \\
\hline TOTAL & 40 & 100,0 & 38 & 100,0 & 19 & 100,0 & 97 & 100,0 & \\
\hline Tipo de pós-graduação & & & & & & & & & \\
\hline Especialização & & & & & & & & & \\
\hline Sim & 26 & 68,4 & 36 & 87,8 & 19 & 100,0 & 81 & 82,7 & $\mathrm{p}^{(1)}=0,006^{*}$ \\
\hline Não & 12 & 31,6 & 5 & 12,2 & - & - & 17 & 17,3 & \\
\hline Mestrado & & & & & & & & & \\
\hline Sim & 4 & 10,5 & 12 & 29,3 & 1 & 5,3 & 17 & 17,3 & $\mathrm{p}^{(1)}=0,027^{*}$ \\
\hline Não & 34 & 89,5 & 29 & 70,7 & 18 & 94,7 & 81 & 82,7 & \\
\hline Doutorado & & & & & & & & & \\
\hline Sim & 11 & 28,9 & 10 & 24,4 & 1 & 5,3 & 22 & 22,4 & $\mathrm{p}^{(1)}=0,120$ \\
\hline Não & 27 & 71,1 & 31 & 75,6 & 18 & 94,7 & 76 & 77,6 & \\
\hline TOTAL & 38 & 100,0 & 41 & 100,0 & 19 & 100,0 & 98 & 100,0 & \\
\hline
\end{tabular}

Área da pós-graduação

Cirurgia bucomaxilofacial

Sim

Não

Ortodontia

Sim

Não

$\begin{array}{lllllllll}15 & 34,9 & 14 & 34,1 & - & - & 29 & 28,2 & \mathrm{p}^{(1)}=0,010^{*} \\ 28 & 65,1 & 27 & 65,9 & 19 & 100,0 & 74 & 71,8 & \\ 7 & 16,3 & 5 & 12,2 & 8 & 42,1 & 20 & 19,4 & \mathrm{p}^{(1)}=0,019^{*} \\ & & & & & & & 80,6 & \end{array}$

Revista da ABENO • 15(3):60-68, 2015. 
Tabela 3 - continuação

\begin{tabular}{|c|c|c|c|c|c|c|c|c|c|}
\hline \multicolumn{10}{|c|}{ Implantodontia } \\
\hline Sim & 4 & 9,3 & 11 & 26,8 & 3 & 15,8 & 18 & 17,5 & \multirow[t]{2}{*}{$\mathrm{p}^{(1)}=0,105$} \\
\hline Não & 39 & 90,7 & 30 & 73,2 & 16 & 84,2 & 85 & 82,5 & \\
\hline \multicolumn{10}{|c|}{ Odontopediatria } \\
\hline Sim & 5 & 11,6 & 4 & 9,8 & - & - & 9 & 8,7 & \multirow[t]{2}{*}{$\mathrm{p}^{(2)}=0,393$} \\
\hline Não & 38 & 88,4 & 37 & 90,2 & 19 & 100,0 & 94 & 91,3 & \\
\hline \multicolumn{10}{|c|}{ Prótese } \\
\hline Sim & 1 & 2,3 & 3 & 7,3 & 5 & 26,3 & 9 & 8,7 & \multirow[t]{2}{*}{$\mathrm{p}^{(2)}=0,014^{*}$} \\
\hline Não & 42 & 97,7 & 38 & 92,7 & 14 & 73,7 & 94 & 91,3 & \\
\hline \multicolumn{10}{|c|}{ Endodontia } \\
\hline Sim & 3 & 7,0 & 3 & 7,3 & 2 & 10,5 & 8 & 7,8 & \multirow[t]{2}{*}{$\mathrm{p}^{(2)}=0,795$} \\
\hline Não & 40 & 93,0 & 38 & 92,7 & 17 & 89,5 & 95 & 92,2 & \\
\hline \multicolumn{10}{|c|}{ Patologia oral } \\
\hline Sim & 4 & 9,3 & 3 & 7,3 & - & - & 7 & 6,8 & \multirow[t]{2}{*}{$\mathrm{p}^{(2)}=0,519$} \\
\hline Não & 39 & 90,7 & 38 & 92,7 & 19 & 100,0 & 96 & 93,2 & \\
\hline \multicolumn{10}{|c|}{ Não sabe ainda } \\
\hline Sim & 9 & 20,9 & 12 & 29,3 & 2 & 10,5 & 23 & 22,3 & \multirow[t]{2}{*}{$\mathrm{p}^{(1)}=0,258$} \\
\hline Não & 34 & 79,1 & 29 & 70,7 & 17 & 89,5 & 80 & 77,7 & \\
\hline TOTAL & 43 & 100,0 & 41 & 100,0 & 19 & 100,0 & 103 & 100,0 & \\
\hline
\end{tabular}

(*): Diferença significativa ao nível de 5,0\%.

(1): Teste Qui-quadrado de Pearson.

(2): Teste Exato de Fisher.

projeto pedagógico destes dois currículos que ora se entremeiam é o aumento de carga horária na atenção básica, via estágio curricular obrigatório.

Neste estudo, a maioria dos entrevistados era jovem, dado que corrobora com achados da literatura pesquisada, o que parece ser também uma tendência nos demais $\operatorname{cursos}^{2,5}$.

No Brasil, segundo o $\mathrm{IBGE}^{6}$, censo demográfico de 2010, a percentagem de mulheres e homens é de $51 \%$ e $49 \%$, respectivamente. Este fato pode justificar a predominância do sexo feminino entre os estudantes do curso de odontologia da UFPE, o que seria apenas reflexo da sociedade ${ }^{2,5,7,8}$.

Em relação ao estado civil, os solteiros perfaziam quase que a totalidade de nossa amostra, concordando com os achados de Rezende et al. $(2007)^{2}$. Este fato também deve ter relação com a pouca idade dos entrevistados ${ }^{5,9}$.

A literatura também corrobora os achados desta pesquisa quanto à identificação com a profissão, traduzida como vocação, como principal resposta do porque da escolha profissional $^{2,5}$.

Não houve predileção quanto ao tipo de trabalho desejado no futuro: iniciativa privada ou setor público. Talvez esta dubiedade se justifique pelo fato da maioria almejar ter as duas situações: um emprego público como garantia de estabilidade e a dedicação ao setor privado para poder alcançar os níveis salariais mais altos. ${ }^{10,11}$

Em alguns trabalhos pesquisados a maioria dos estudantes pertencia à classe alta, ${ }^{5}$ com média de renda mensal superior a 30 salários mínimos ${ }^{2}$, enquanto que no presente estudo mais de $50 \%$ dos alunos relatou renda mensal compatível com padrão de classe média.

O curso de especialização como forma de pós-graduação foi a alternativa mais escolhida entre os entrevistados, tendo como principais especialidades a cirurgia, implantodontia e ortodontia. Resultados que se aproximam dos encontrados na literatura ${ }^{2,5,12}$. Contudo, a maior parte dos entrevistados afirma querer trabalhar no setor público, em que a estratégia de saúde da família é a principal 
oferta de empregos e não absorve profissionais com especialidades nestas áreas. A escolha por estas áreas pode também ser justificada pelo fato de que, culturalmente, existe o ideário de que sejam especialidades mais rentáveis do ponto de vista econômico. A pretensão salarial inicial também corrobora com os achados na literatura ${ }^{2,5}$.

A Inclusão do estudante de odontologia da UFPE na rede do Sistema Único de Saúde é feita deste o inicio da graduação, pelo PETSAÚDE (Programa de Educação pelo Trabalho para a Saúde), instituído em 2008 pela Portaria Interministerial MS/MEC $\mathrm{n}^{\mathrm{o}} .1 .802 / 08^{13}$ e tem como objetivo permitir ao estudante vivenciar, durante a graduação, o funcionamento das Unidades de saúde da Família e a importância da promoção de saúde na atenção primária no contexto da realidade social. Este dado pode justificar a razão dos estudantes terem avaliado a "capacidade de promover saúde" como o principal fator de realização profissional.

Diante deste panorama, são necessários estudos futuros mais específicos e abrangentes, para determinar o impacto da mudança curricular e do projeto pedagógico do curso nas expectativas dos graduandos.

\section{CONCLUSÃO}

O perfil dos estudantes entrevistados foi formado por uma maioria jovem, solteira e do sexo feminino e pertencentes à classe média. A identificação com a profissão se configurou no principal motivo pela escolha da odontologia, sendo reconhecido o padrão de qualidade das Instituições Federais de Ensino Superior. O mercado de trabalho parece ser uma opção animadora tanto no âmbito do serviço público como no setor privado, sendo a promoção de saúde considerada a principal fonte de realização profissional. A estimativa inicial de renda está dentro dos limites de renda familiar atual dos entrevistados. Cursos de pósgraduação nas especialidades de cirurgia bucomaxilofacial, ortodontia e implantodontia são os mais desejados.

\section{REFERÊNCIAS}

1. Teixeira MAP, Gomes WB. Estou me Formando... E Agora? Reflexões e perspectivas de jovens formandos universitários. Rev Bras Orientac Prof 2004;5:47-62.

2. Rezende FP, Nakanishi FC, Machado ACP, Quirino MRS, Anbinder AL. Perfil, motivações e expectativas dos graduandos e graduados em odontologia. Rev odontol Univ Cid São Paulo. 2007;19:165-72.

3. Unfer BR, Rigodanzo L, Hahn D, Manfredini D, Rodrigues E, Cavalheiro $\mathrm{CH}$. Expectativas dos acadêmicos de odontologia quanto a formação e futura profissão. Saúde 2004;30:33-40.

4. Melo TAF, Kunert GG, Limongi O, Matos APG, Almeida V. Centro de Especialidades Odontológicas (CEO): uma avaliação qualitativa na perspectiva dos alunos participantes. Stomatos 2009;15:32-7.

5. Junqueira JC, Colombo CED, Tavares PG, Rocha RF, Carvalho YR, Rodrigues JR. What thinks and who is the dentistry undergraduate. Rev Odontol UNESP 2002;31:269-84.

6. IBGE. [Internet]. Brasília: Brasil tem mai mulheres. 2013. Disponível em: http://7a12.i bge.gov.br/voce-sabia/curiosidades/brasil-temmais-mulheres).

7. Costa SM, Durães SJA, Abreu MHNG. Feminização do curso de odontologia da Universidade Estadual de Montes Claros. Ciênc Saúde Coletiva 2010;15:1865-73.

8. Morita MC, Haddad AE, Araujo ME. Perfil atual e tendências do cirurgião-dentista brasileiro. Maringá: Dental Press International; 2010.

9. Loffredo LCM, Pinelli C, Garcia PPNS, Scaf G, Camparis CM. Socioeconomic, cultural and familiar characteristics of dental students. Rev Odontol UNESP 2004; 33:175-82.

10.Hoeppner MG, Izzo CBA, Araújo CSA, Prandi LR. Avaliação da expectativa profissional de 
acadêmicos do curso de Odontologia da Unipar - Umuaama, PR. EDU Rev Educ 2005;5:111-9.

11. Machado FC, Souto DMA, Freitas CHSM, Forte FDS. Odontologia como escolha: perfil de graduandos e perspectiva para o futuro profissional. Rev ABENO 2010;10:27-34.

12. Toassi RFC, Souza JM, Rösing CK, Baumgarten A. Perfil sociodemográfico e perspectivas em relação à profissão do estudante de odontologia da Universidade Federal do Rio Grande do Sul, Brasil. Rev Fac Odontol Porto Alegre. 2011;52:25-32.

13.PET-Saúde. Brasília: Ministério da Saúde; 2011. Disponível em: http://portal.saude.gov. $\mathrm{br} /$ portal/saude/profissional/visualizar texto.c fm?idtxt $=35306$

\section{ABSTRACT}

Dental student's expectations regarding their professional future in dentistry

This study aimed to know the expectations, professional projects and projections of success in the work activities of dental students from the Federal University of Pernambuco. The study population consisted of dental students from $1^{\text {st }}$ daytime and nighttime periods $(\mathrm{N}=43), 5^{\text {th }}$ period $(\mathrm{N}=41)$ and $10^{\text {th }}$ period $(\mathrm{N}=21)$. Most students interviewed were young, unmarried (99\%) and female (65.7\%), having a family income of up to $\mathrm{R} \$ 5,100.00$ per month $(80.9 \%)$. We found that $83.8 \%$ of them chose to study dentistry because they felt identified with the profession. Students also recognized the quality standard of Federal Institutions of Higher Education and this was the reason for choosing the university $(74.3 \%)$. There were no differences regarding to join the public or private labor markets in the future, being both viable options. Health promotion was reported as the main source of job satisfaction (71.4\%), especially for students from early periods of the course $(p=0.026)$. The initial estimate of income is within the limits of current family income for the majority of respondents (59\%). Of respondents, $98.1 \%$ plan to join a postgraduate course and specializations was the most common goal (78.6\%). Specialties such oral and maxillofacial surgery, orthodontics and implantology are most sought.

Descriptors: Evaluation. Education, Higher. Dentistry.

Correspondência para:

Profa. Elaine Judite de Amorim Carvalho

e-mail: elaine_judite@yahoo.es

Rua Quimera. Q-D4. N² 29. Ouro Preto

52370-290 - Olinda - Pernambuco 